\title{
Research on wireless sensor network middleware technology for live detecting of substation equipment
}

\author{
Hai $\mathrm{Yu}^{1}$, Lin Peng ${ }^{1}$, He Wang ${ }^{1}$, Hui Deng ${ }^{1}$ and Chang-Fu Xu ${ }^{2}$ \\ ${ }^{1}$ Global Energy Interconnection Research Institute, Nanjing, Jiangsu, China \\ ${ }^{2}$ Jiangsu Electric Power Company Research Institute, Nanjing, Jiangsu, China \\ E-mail:yuhai@geiri.sgcc.com.cn
}

\begin{abstract}
In substation equipment charged detection wireless sensor network level, fault prevention and fault occur in the repair system, in all stages of the life cycle of the network, including testing, deployment and maintenance, the need for a lot of human-computer interactive operation. Different operation requirements of sensor nodes have different behavior to match, and some of them are conflicting. In order to solve this problem, through abstract behavior in the life cycle of a human-computer interactive operation, the introduction of mode concept, and the concept, design of the behavior mode of wireless sensor network middleware provide the application program organization and switching strategy, services and modules, which can effectively improve the node software development efficiency and scalability.
\end{abstract}

Keywords: Substation Equipment Live Detection; Behavior Pattern; Network Middleware.

\section{Introduction}

Based on the wireless sensor network, the field application of the charging detection device of the substation equipment can significantly improve the efficiency of the charged detection work, and it is the development trend of the live line detection technology. Due to the substation deployed in harsh environments, the node capacity constraints, sensor network maintenance is a major challenge, researchers at home and abroad have been for the particular stage of the life cycle of the wireless sensor network system specific humanmachine interaction problem proposed multiple solutions. In practical applications, systems are often required to support the operation of all phases of the life cycle. However, the demand for node behavior exists huge difference, how to support these operations all application reasonable organization in a sensor node software, according to the stages of the life cycle of conversion 
switch application is running, adjust the characteristics of node behavior, improving the operation efficiency of the nodes, there is a lack of such research.

The existing wireless sensor network operating system does not provide relevant support, the developer must design the application of the organization and switching strategy. Due to the diversity of the wireless sensor network applications, such as for different applications are designed for different application program organization and switching strategy, node software limits scalability and reusability, greatly increasing the difficulty of development. Therefore, it is needed to implement the organization and switching of the multi behavior applications of the system maintainability by constructing the middleware between the application and the operating system.

\section{Wireless Sensor Network Middleware Technology}

Using middleware technology realize sensor node application and the underlying support for the separation, convenient application development, expansion and transplantation and node performance optimization has become one of the hot field in the research of wireless sensor network. However, there is still a lack of relevant discussion on the organization and handover mechanism of multi behavior applications in the aspect of system maintainability.

The COUGAR[1], TinyDB[2] and SINA[3] and other wireless sensor network middleware to wireless sensor network as for distributed database, provides a similar to the syntax of SQL to query the data in wireless sensor networks; tinylime is a tuple space based Middleware for wireless sensor networks, applications can be from a shared tuple space using insert and remove commands; mires is a publish / subscribe to the solution, the operating system for wireless sensor network events provides further support.

In this paper, the design of wireless sensor network middleware is based on virtual machine. Currently such middleware is mainly from the node function expandability of, such as mate[4] by using simple and lightweight virtual machine instruction set for the expression of complex applications, reducing the volume of applications, thereby reducing the software update required communication energy overhead. Davim[5] also based on the idea of virtual machine. The main to simple function of the software reuse, separation based services and applications, support in the independent space concurrently running applications and dynamic management of available services as the goal by in the virtual machine in the concurrent execution of applications, application is completely separated, and supports at runtime as nodes join new application features. Different from the above work, this paper research priorities for the 
wireless sensor network middleware is on system behavior of different applications based on human-computer interaction and data acquisition operations, changes in demand for rational organization and switching and management, effectively improve the maintainability of the system.

\section{Wireless Sensor Network Middleware Based on Behavior Pattern}

\subsection{System maintainability function analysis}

The typical human computer interaction operation in the life cycle of the sensor network system is analyzed, and the maintenance requirements of the system are summarized:

The local hardware detection, in the development / testing stage hardware function test is helpful to prevent the failure of node deployment in wireless system; code distribution system should support, through the wireless channel will be distributed to the node software code one-time multiple or even all of the sensor nodes in the network, thereby greatly improving the operation efficiency, this feature can also be used system maintenance,; wireless node configuration, site node parameters re configuration can improve the flexibility of the system in different deployment environment; on-line sensor calibration, during the deployment phase in while the need for sensors to calibration, and in the maintenance phase also requires periodic calibration of sensor; remote fault detection system, to reduce the maintenance cost it should be on a remote base station system fault detection, periodically obtaining system state and report to the remote distance and background; Field system fault diagnosis, in the detection of system failure, remote diagnosis can be initially inferred fault causes.

In the life cycle of wireless sensor network, with the change of the stage, the man-machine interactive operation requirement will change, and the sensor nodes need to be switched to run the application program to perform different functions. There is a big difference between these applications in the demand for the behavior characteristics of nodes, and there are some similarity in behavior.

\subsection{Middleware design}

In order to realize the management of universal and extensible work mode, this paper designs a wireless sensor network middleware based on behavior mode. In the middleware, the work mode is used as an abstract container of the application, and the application program with the same behavior characteristic is stored in the same working mode. In order to meet the changing of human- 
computer interaction and data acquisition operations demand and need to design operating mode management strategy, to switch the operating mode, operation and hang belongs to the work mode of application, and the allocation of resources to change the node behavior. At the same time, to support the function of the application program with many of the same basic logic functions, such as local hardware detection, on-site system fault diagnosis, online sensor calibration are required to sample the sensor data; and wireless code distribution, wireless node configuration are required to read and write the external memory. Therefore, it is necessary to abstract the common function of the application program, which is designed to be independent of the working mode of the general purpose logic supply procedure call, so as to reduce the coupling degree of the program. Design focus mainly includes working mode, working mode management general logic.

\subsubsection{Middleware architecture}

The architecture of the middleware is shown in figure 1. The common logic library comprises a plurality of working modes, a work mode management unit, a function reuse, and a plurality of application programs. The working mode of the middleware is divided into two categories: basic work mode and custom work mode. Support the typical system maintenance functions and data acquisition functions for basic operation modes, as a core component of the middleware; custom mode is according to the demand of application in the node software development add and settings to support more system maintenance functions. The two modes of operation provide a unified operation interface for the application to register to a working mode or delete from the working mode, and use a unified work mode management strategy. The work mode management unit accepts the new working mode or deletes the original working mode through the mode register / delete module. In operation process, mode switching management module will according to the current human-computer interaction operation and data acquisition needs, in registered mode switch to run the appropriate application, and the model of resource management module called lower network protocol and distribution hardware resource, to modify the behavior of a node. 


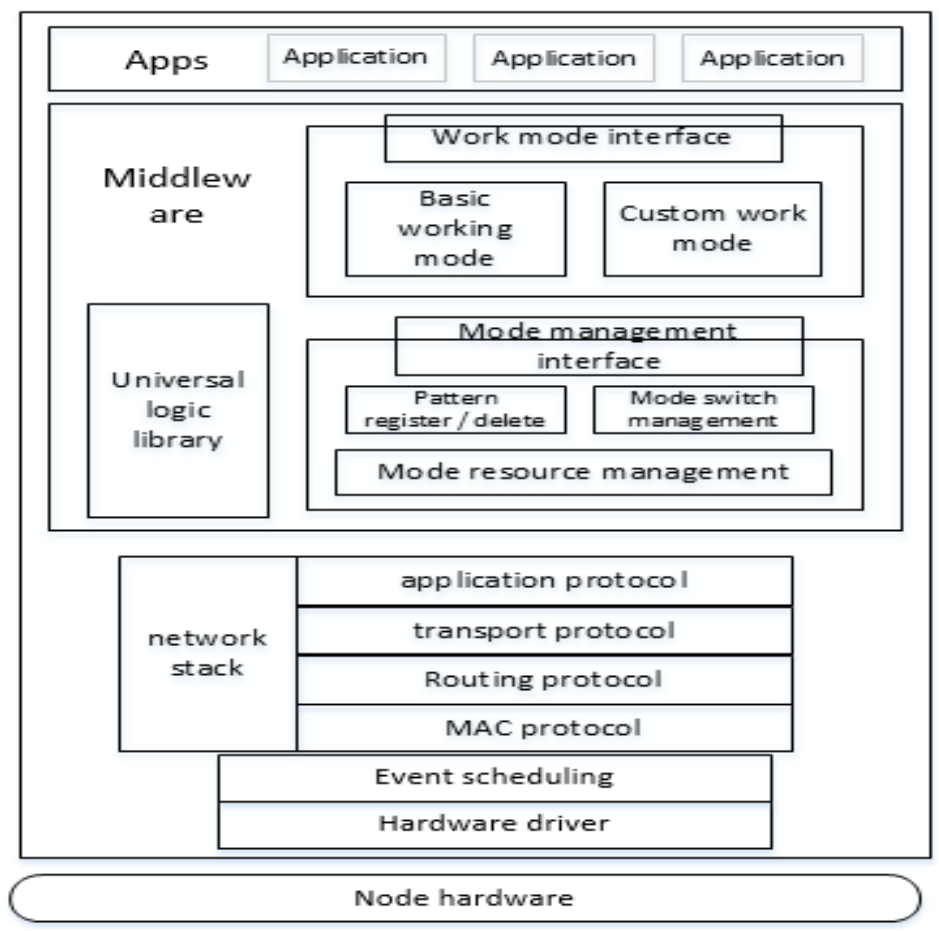

Fig. 1 Schematic diagram of the middleware architecture

\subsubsection{Basic work mode design}

According to the described in section 2-1 typical system can maintain functional behavior, the behavior pattern classification established five basic modes: normal operation mode, network test mode, local access pattern, the code update mode and the factory test mode. In each mode, one or more of the applications can be executed concurrently to achieve the desired functionality.

\subsubsection{Universal logic library}

Universal logic library provides the basic function logic of common operation in wireless sensor networks, which can simplify the system development and reduce the coupling degree. Each logical module in the library provides access to the interface, and implements the specific function logic. Through analysis of typical wireless sensor network applications, middleware design basic universal logic modules, including: node sleep / wake, battery voltage acquisition, access to peripheral status, access to peripheral state test, sensor, sampling sensor, external memory accessing. 
Node sleep / wake-up universal logic is mainly used to meet the normal operation and network test mode to reduce power consumption, the logic mainly provides micro controller nodes and all the peripheral hardware devices opening / closing operation interface. The voltage of the battery and peripheral status to obtain universal logic are needed to understand the current battery level and peripheral status of application, such as running status report, check node, node self checking provide a uniform access interface. Peripheral status testing universal logic contains a series of test logic, each logic can be tested on the specified peripheral hardware devices tested and returns the result of the test, to support the node checks the application user assisted diagnosis of nodes on a specific device, and to support the node testing program automatically complete all the hardware test. Sensor state acquisition and sensor sampling general logic provides a unified sensor access interface, thus shielding the upper layer application of different sensors operating interface and instructions. External memory access general logic through the file system in the form of external storage space organization, and provide a unified read / write interface.

\section{Conclusion}

In this paper, we study the efficient organization and switching method of multi behavior applications in wireless sensor networks from the point of view of the middleware. Based on the experience of field development, deployment and maintenance, the interaction and similarity of typical human computer interaction features in all stages of the system life cycle are discussed and abstracted. On this basis, the concept of operation mode is introduced as a container with similar behavior characteristics. Then, a behavior model based Middleware for wireless sensor networks is proposed. It can effectively improve the development efficiency and scalability of the node software by organizing and switching the basic policies, services and modules provided by the middleware. Variable electrical detection of wireless sensor network field using middleware, system testing and deployment time efficiency improves the 6 times, and through the system fault detection and diagnosis function, system has been the scene of the wireless sensor network field operations personnel maintenance, greatly reducing the few experts to the scene to maintain the system bring great manpower and time cost. 


\section{Acknowledgments}

Thanks for the support of the science and technology project to State Grid Corporation "Research and pilot application of power grid equipment state intelligent sensing technology based on Internet of things".

\section{References}

1. Bonnet P, Gehrke J, Seshadri P. Towards sensor database systems. Lecture Notes in Computer Science, 2001. pp. 3-14.

2. Madden S R, Franklin M J, Hellerstein J M. et a1. Tinydb: an acquisitional query processing system for sensor networks. ACM Transactions on Database Systems, 2005, 30(1): pp. 122-173.

3. Shen C C, Srisathapomphat C, Jaikaeo C. Sensor information networking architecture and applications. IEEE Personal Communications, 2001, 8(4): pp. 52-59.

4. Levis P, Culler D. Mate: a tiny virtual machine for sensor networks. ACM SIGARCH Computer Architecture News, 2002, 30(5): pp. 85-95.

5. Horre W, Michiels S, Joosen W, et a1. Davim: adaptable middleware for sensor networks. IEEE Distributed Systems Online, 2008, 9(1): pp. 1-11. 